\title{
How we do it: the Running-X suture technique
}

\author{
Nathaniel L. Villanueva ${ }^{1}$, Kyle Sanniec ${ }^{1}$, Ronald Mancini ${ }^{2}$ \\ ${ }^{1}$ Department of Plastic Surgery, University of Texas Southwestern Medical Center, Dallas, TX 75390, USA. \\ ${ }^{2}$ Oculoplastic and Orbital Surgery, Department of Ophthalmology, University of Texas Southwestern Medical Center, Dallas, TX \\ 75390, USA.
}

Address for correspondence: Dr. Ronald Mancini, Oculoplastic and Orbital Surgery, Department of Ophthalmology, University of Texas Southwestern Medical Center, 5323 Harry Hines Blvd., Dallas, TX 75390, USA. E-mail: ronald.mancini@utsouthwestern.edu

\begin{abstract}
There are a myriad of suture techniques available to close incisions of the brow and forehead, each with their own advantages and disadvantages. The ideal suture technique would provide excellent cosmetic results, offer expedient wound closure, optimize skin eversion and wound edge apposition, and provide excellent cosmetic results. The authors describe a new suture technique, the Running- $X$ suture, a running horizontal mattress suture that has successfully been used by the senior author for many years to re-approximate surgical wounds of the brow and forehead in an expeditious and aesthetic manner.
\end{abstract}

Key words:

Suture technique; running horizontal mattress suture; forehead incisions; eyebrow incisions

\section{INTRODUCTION}

The choice of suture technique used to close incisions depends on multiple variables which include anatomic location, thickness of skin, type of wound, and degree of tension. Of the various epidermal skin closure techniques used throughout the body, simple interrupted and simple running sutures are the most common because of the ease of placement and speed of closure. ${ }^{[1,2]}$ Although simple interrupted sutures are easy to place and have a lower potential to cause impaired cutaneous circulation, the major disadvantages are that the technique is more time consuming and produces minimal eversion. The main advantages of using a simple running suture are the rapid closure of wounds and its ability to simultaneously approximate the dermis and epidermis. However, its major disadvantage is poor control of inversion and eversion of the epidermal edges. Mattress sutures are

\begin{tabular}{|l|l|}
\hline \multicolumn{2}{|c|}{ Access this article online } \\
\hline Quick Response Code: & Website: \\
\hline & http://www.parjournal.net \\
\cline { 2 - 3 } & \\
\hline $\mathbf{D}$ & DOI: \\
\hline
\end{tabular}

also widely used in epidermal closures because of their ability to produce eversion, compression of wound edges, and close wounds under moderate tension. ${ }^{[3,4]}$ One of the major disadvantages to mattress sutures, both vertical and horizontal, is they are more prone to become buried during the healing process, requiring a more tedious removal. Combining the advantages of mattress sutures with the time saved using a running technique has been widely described, although the wound edge apposition tends to be imprecise. ${ }^{[2]}$

Herein, we describe the Running-X suture, a novel running horizontal mattress suture. This suture technique provides multiple advantages when compared to traditional running mattress sutures. The Running-X

This is an open access article distributed under the terms of the Creative Commons Attribution-NonCommercial-ShareAlike 3.0 License, which allows others to remix, tweak and build upon the work non-commercially, as long as the author is credited and the new creations are licensed under the identical terms.

For reprints contact: service@oaepublish.com

How to cite this article: Villanueva NL, Sanniec K, Mancini R. How we do it: the Running-X suture technique. Plast Aesthet Res 2016;3:245-7.

Received: 20-05-2015; Accepted: 30-03-2016 
provides rapid wound closure, excellent skin eversion, precise wound edge apposition, ability to close wounds under tension, ease of suture removal, and most importantly excellent cosmesis in sensitive areas of the face such as the temporal brow and forehead.

\section{TECHNIQUE}

\section{Anchoring stitch}

The Running-X begins with a simple interrupted stitch. The initial throw is passed from the epidermis on the opposite side of the wound, through the wound, and then out of the epidermis. A knot is tied and the free end of the suture tail is cut leaving a small tail [Figure 1a].

\section{Running-X}

The needle is then picked up, reloaded and inserted in the epidermis on the opposite side of the anchoring knot approximately $4 \mathrm{~mm}$ from the initial stitch (far) and $2 \mathrm{~mm}$ from the wound edge. The needle is passed perpendicular to the wound edge, across the wound and through the epidermis on the same side as the anchoring knot [Figure 1a]. The suture is pulled through leaving a small loop between the anchoring knot and the entry point of the first throw. The needle is then thrown at the midpoint between the first throw and the anchoring stitch (near), inside the loop, starting on the opposite side of the anchoring knot $2 \mathrm{~mm}$ from the wound edge, with the needle passed perpendicularly though the wound, and ending through the epidermis on the same side as the anchoring knot [Figure $1 \mathrm{~b}]$. The next throw is then started $4 \mathrm{~mm}$ from the first far throw starting on the opposite side of the anchoring knot [Figure 1c]. This will now produce what appears to be a "double-X" pattern over the wound. Following the second far throw, the next throw is placed midway between the first and second far throws inside the loop between the first near throw and the second far throw making this the second near throw [Figure 1d]. This pattern of far-near is repeated for the remainder of the wound.

\section{End stich}

After the Running-X is used across the entire wound length, the suture is tied off using a final far throw. This final far throw is different from the previous far throws because it is placed only $2 \mathrm{~mm}$ from the previous far throw as opposed to $4 \mathrm{~mm}$ like the previous ones [Figure 1e]. The suture is secured with a knot created using the loop between the previous near exit site and entry of the final far throw, and the end of the suture. The tails are then cut short.

\section{Suture material}

We prefer to use polypropylene (Prolene, Ethicon, Somerville, NJ, USA). This suture material provides more elasticity and stretch than nylon. This is important because it allows for wound edema and decreases the risk of tissue strangulation and necrosis..$^{[3]}$

\section{Time to removal}

The Running-X suture is removed at the appropriate time interval for the specific anatomic locations to avoid track
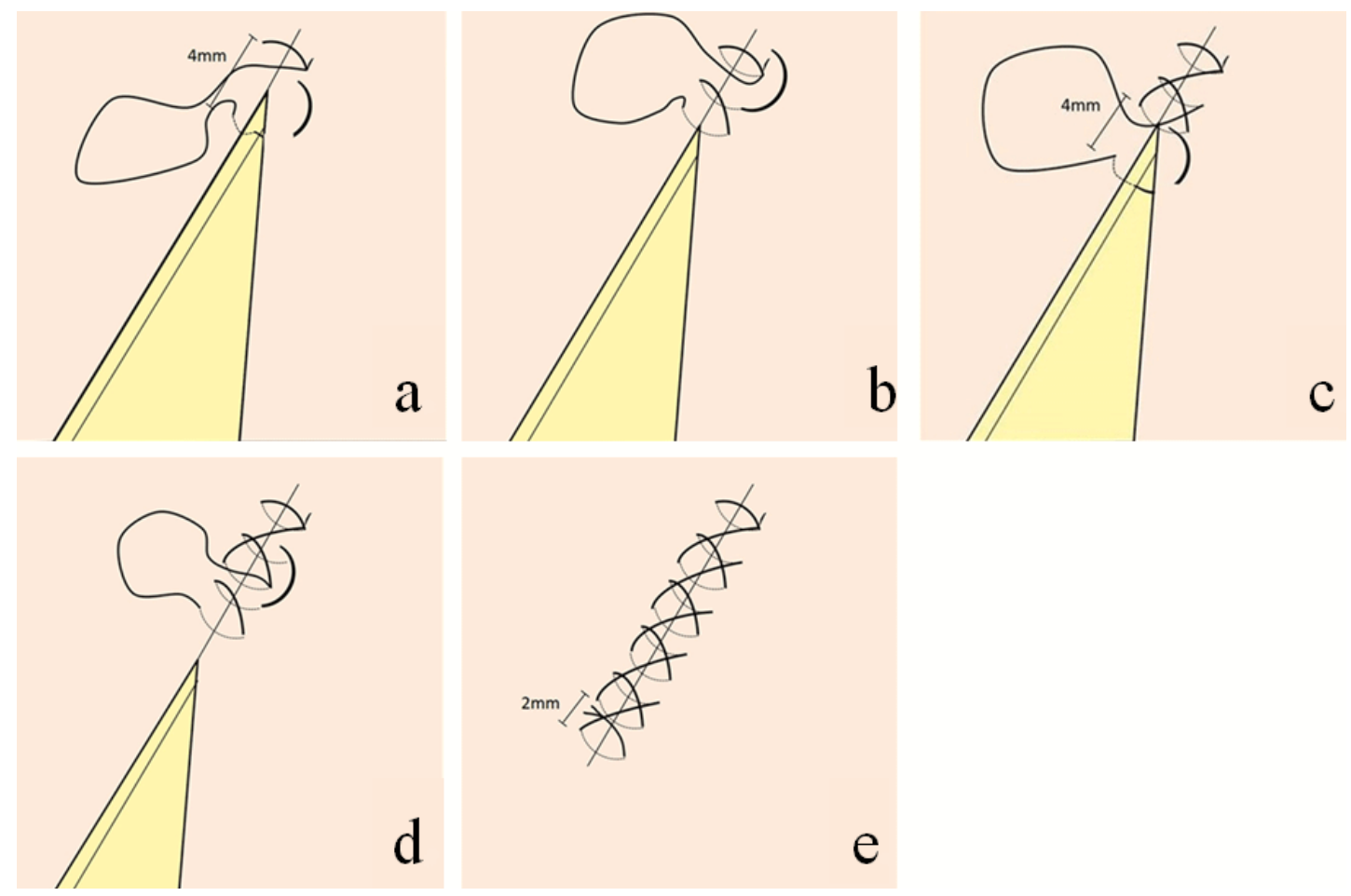

Figure 1: Running-X suture technique. (a) Anchor stitch and first far throw; (b) first near throw midway between anchor stitch and far throw; (c) second far throw $4 \mathrm{~mm}$ distal to first far throw; (d) second near throw midway between first and second far throw; (e) final far throw only $2 \mathrm{~mm}$ distal to penultimate far throw and secured to loop between the last near and last far throw with tails cut 

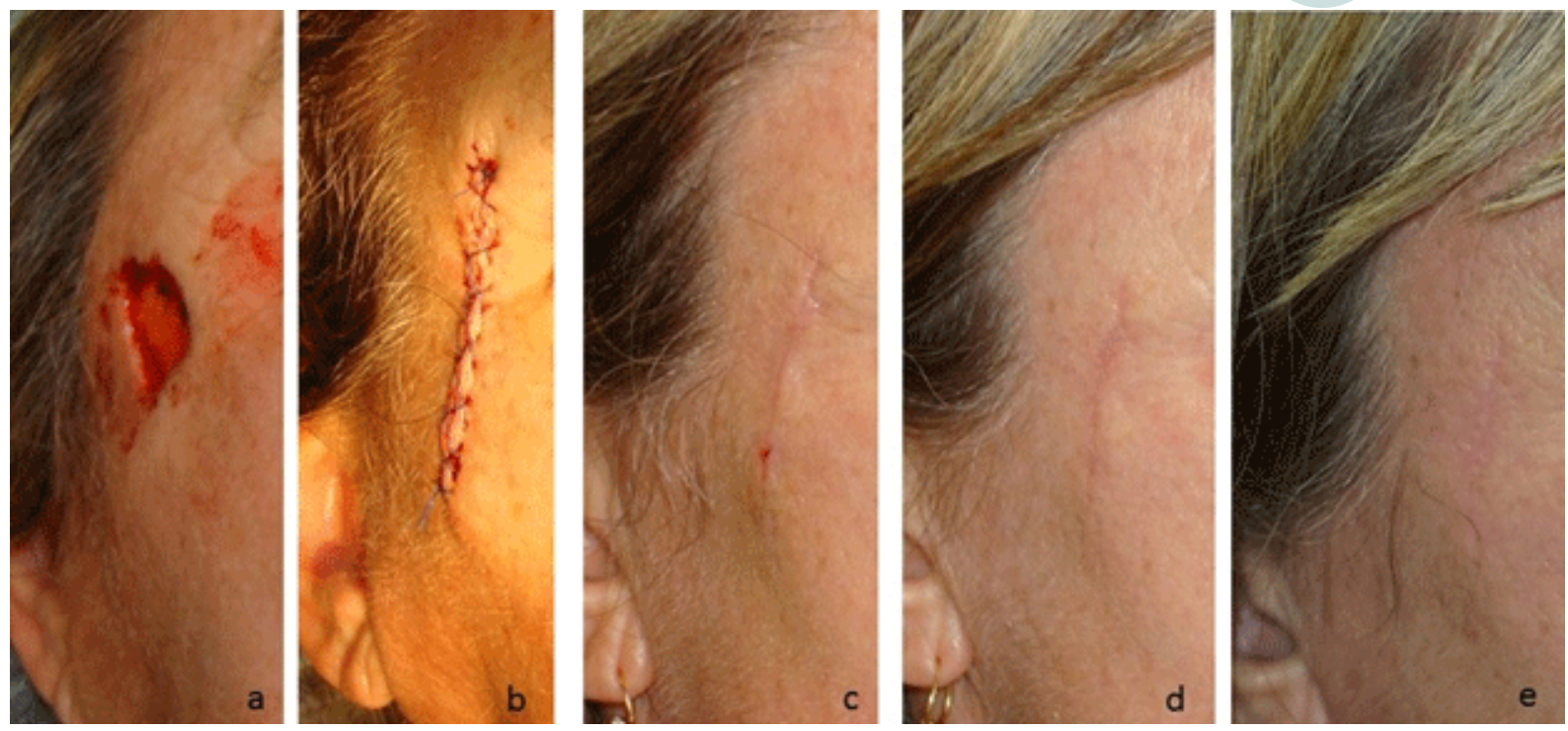

Figure 2: (a) Preoperative defect of the right temporal area following Mohs surgical excision of a Basal cell carcinoma, in a 58-year-old female with Fitzpatrick II skin type; (b) intraoperative appearance following dog ear excision and closure using the Running-X technique; (c) ten days postoperatively; (d) six weeks postoperatively; (e) twelve weeks postoperatively

marks. The authors in this report have primarily used this technique for brow and forehead wound closures. Therefore, the sutures were removed at 5 to 7 days from closure.

\section{DISCUSSION}

The senior author (Ronald Mancini) has successfully used this technique for many years to re-approximate surgical wounds of the brow, forehead and temporal area. Since the Running- $\mathrm{X}$ technique is continuous it allows for rapid wound closure. The needle is always thrown in the same direction, reducing the time to reload the needle compared to a running horizontal mattress suture which needs to be reloaded in opposing directions with each throw. This technique functions as a horizontal mattress specifically at the interval between the far and near throws. The horizontal and oblique forces placed across the wound at these intervals of the technique provide excellent skin eversion and precise wound edge apposition. The eversion is created in a similar fashion to running horizontal mattress sutures. However, it is superior to the running horizontal mattress because the "X"s created over the wound edges provide a leveling force for the epidermal edges. In addition, the Running-X is excellent for closure of wounds under tension because it provides added strength. A similar suture pattern has been described for epitendinous suture in tendon repairs, and when compared to a simple running suture, the similar patterned suture provided a $245 \%$ increase in tensile strength. ${ }^{|5|}$ Since this technique places suture strands over the wound, unlike traditional running mattress sutures, these strands can easily be divided at the time of suture removal with minimal patient discomfort. Finally, through a summation of the advantages of the
Running-X, this technique has provided the authors with excellent cosmetic results [Figure 2].

We do not recommend this suture technique for anatomic locations with thinner, fragile skin or distorted wound edges due to the increased risk of tissue strangulation and wound dehiscence. We also recommend against over tightening this suture in order to avoid tissue strangulation.

Despite our positive clinical experience with this technique, further studies are required to further define the limitations and tissue biomechanics of this technique and a prospective study comparing the Running-X suture technique with commonly used running and interrupted suture techniques is necessary before any definitive conclusions can be drawn.

\section{Financial support and sponsorship} Nil.

\section{Conflicts of interest}

There are no conflicts of interest.

\section{REFERENCES}

I. Adams B, Levy R, Rademaker AE, Goldberg LH, Alam M. Frequency of use of suturing and repair techniques preferred by dermatologic surgeons. Dermatol Surg 2006;32:682-9.

2. Wong NL. Review of continuous sutures in dermatologic surgery. J Dermatol Surg Oncol 1993; 19:923-31.

3. Krunic AL, Weitzul S, Taylor RS. Running combined simple and vertical mattress suture: a rapid skin-everting stitch. Dermatol Surg 2005; 31:1325-9.

4. Moy RL, Lee A, Zalka A. Commonly used suturing techniques in skin surgery. Am Fam Physician 1991;44:1625-34.

5. Kim PT, Aoki M, Tokita F, Ishii S. Tensile strength of cross-stitch epitenon suture.J Hand Surg Br 1996;21:821-3. 